\title{
Comparación de hemodiafiltración "mid-dilucional" respecto a hemodiafiltración pre y postdilucional
}

\author{
Premio Fresenius Medical Care
}

\author{
Magda Sánchez - Ma Rosa Vallvé - Mª Teresa López - Neus Gispert - Ángeles Mayordomo \\ Sonia Lage - Antonia Vives
}

Unidad Diálisis del Hospital Clínic. Barcelona

\begin{abstract}
Resumen
La hemodiafiltración permite varias alternativas según se incorpore el líquido de reinfusión: pre-dilucional (antes del dializador), post-dilucional (después del dializador) y mixta (pre y post-dilución). Una alternativa a esta última es un dializador diseñado para realizar la mid-dilución, dónde la sangre entra por un haz de fibras centrales y regresa, en sentido contrario, por fibras periféricas. El líquido de reinfusión se incorpora en la mitad de los dos tramos del dializador, así en el primer tramo se produce una hemodiafiltración post-dilucional y en el segundo tramo una hemodiafiltración pre-dilucional.
\end{abstract}

El objetivo de este estudio fue comparar el modo de infusión mid-dilucional con los modos pre y post-dilucional, evaluando la depuración de varias moléculas de diferente peso molecular.

Cada paciente fue sometido a tres sesiones de diálisis con una técnica diferente cada vez, respetando las características de la diálisis a excepción del volumen de infusión.

La hemodiafiltración on-line con el modo de infusión mid-dilucional parece ser una buena alternativa que permite altos volúmenes convectivos con una mayor depuración de $\beta 2$-microglobulina, prolactina, mioglobina y proteína transportadora de

Correspondencia:

Magda Sánchez

Santa Carolina 30, ático $1^{\text {a }}$

08025 Barcelona

magdasanruiz@hotmail.com retinol en comparación con el modo postdilucional, y más Ilamativa en comparación con la hemodiafiltración predilucional.

\section{PALABRAS CLAVE:}

- HEMODIAFILTRACIÓN MID-DILUCIONAL

- HEMODIAFILTRACIÓN ON-LINE

- EFICACIA DIALÍTICA

\section{Comparison of "mid-dilution" haemodiafiltra- tion with pre- and post-dilution haemodiafiltra- tion}

\section{Abstract}

Various alternative forms of haemodiafiltration are possible depending on when the reinfusion liquid is incorporated: pre-dilution (before the dialyser), postdilution (after the dialyser) and mixed (pre- and post-dilution). An alternative to the latter is a dialyser designed for mid-dilution, where the blood passes through a membrane of central fibres and returns, in the opposite direction, through peripheral fibres. The reinfusion liquid is incorporated in half of the two sections of the dialyser. Thus, in the first section post-dilution haemodiafiltration takes place, and in the second section, pre-dilution haemodiafiltration.

The aim of this study was to compare the forms of mid-dilution infusion with pre-and post-dilution, evaluating the removal of various molecules with different molecular weight.

Each patient underwent three dialysis sessions, using a different technique each time, respecting 
the characteristics of the dialysis except for the infusion volume.

Mid-dilution on-line haemodiafiltration seems to a be a good alternative that permits high convective volumes with a greater purification of $\beta 2$-microglobulin, prolactin, myoglobin and retinol binding protein compared to post-dilution haemodiafiltration, and more marked compared to pre-dilution haemodiafiltration.

\section{KEY WORDS:}

- MID-DILUTION HAEMODIAFILTRATION

- ON-LINE HAEMODIAFILTRATION

- DIALYTIC EFFICACY

\section{Introducción}

La hemodiálisis se puede considerar una terapia sustitutiva renal que garantiza resultados a corto plazo razonables. Sin embargo, los resultados clínicos a largo plazo podrían ser mejorados. Muchas de las complicaciones de los pacientes en hemodiálisis han sido atribuidas a una incompleta depuración de solutos potencialmente dializables y a la acumulación de solutos de gran peso molecular que son difíciles de eliminar por diálisis convencional.

La hemodiafiltración en línea (HDF on-line) es una de las técnicas más recientes de hemodiálisis. Su característica principal es que el propio monitor de diálisis genera el líquido de sustitución de manera continua a partir del líquido de diálisis ${ }^{1}$. En la hemodiafiltración se utilizan con gran eficacia el transporte difusivo y el convectivo. Esta técnica precisa de dializadores de alto flujo, membranas de alta biocompatibilidad, líquido de diálisis ultrapuro y altos volúmenes de reposición (entre 5 y $10 \mathrm{~L} / \mathrm{h}$ ). Los elevados volúmenes de reposición proporcionan una manera óptima de eliminar toxinas urémicas y depuran de forma muy eficiente las pequeñas y medianas moléculas sin que exista retrofiltración ${ }^{2}$.

La HDF permite varias alternativas según se incorpore el líquido de reinfusión: pre-dilucional (antes del dializador), post-dilucional (después del dializador) y mixta (pre y post-dilución) ${ }^{3}$.
- Hemodiafiltración post-dilucional. Es el método de infusión más eficiente para obtener aclaramientos de pequeños solutos y sustancias de mayor peso molecular. Este método puede provocar complicaciones técnicas, como la hemoconcentración o elevación de la presión transmembrana (flujos de ultrafiltración limitados).

- Hemodiafiltración pre-dilucional. Respecto a la hemodiálisis de alto flujo, presenta unos aclaramientos más bajos de solutos de pequeño peso molecular, aunque permite una mejor extracción de medias y altas moléculas. Se puede utilizar con elevados flujos de infusión y reduce la hemoconcentración.

- Hemodiafiltración mixta (pre- y post-dilucional simultáneamente). Esta podría ser una técnica altamente eficaz para depurar toxinas urémicas, a la vez que elude las desventajas de los dos métodos anteriores. Una alternativa reciente a este método es un dializador diseñado para realizar la mid-dilución, dónde la sangre entra por un haz de fibras centrales y regresa, en sentido contrario, por fibras periféricas. El líquido de reinfusión se incorpora en la mitad de los dos tramos del dializador, así en el primer tramo se produce una hemodiafiltración post-dilucional y en el segundo tramo una hemodiafiltración pre-dilucional.

\section{Objetivo}

El objetivo de este estudio fue comparar, con la misma membrana de diálisis, el modo de infusión mid-dilucional con los modos pre y post-dilucional, evaluando la depuración de varias moléculas de diferente peso molecular.

\section{Material y Métodos}

Este estudio prospectivo fue realizado en 20 pacientes ( 12 hombres y 8 mujeres) con una edad media de $57,8 \pm 18$ en programa estable de hemodiálisis y con un buen acceso vascular.

Cada paciente fue sometido a tres sesiones de diálisis: una con membranas de polifenileno de 1,7 $\mathrm{m}^{2}$ y $200 \mathrm{~mL} / \mathrm{min}$ de infusión pre-dilucional, otra con membrana de polifenileno de $1,7 \mathrm{~m}^{2}$ y $100 \mathrm{~mL} / \mathrm{min}$ 
de infusión post-dilucional, y una tercera con una membrana de polifenileno de $1,9 \mathrm{~m}^{2}$ y $200 \mathrm{~mL} / \mathrm{min}$ de infusión mid-dilucional. Se randomizó el orden de las tres sesiones.

El resto de parámetros de diálisis no variaron: monitor Fresenius $4008 \mathrm{~S}$, flujos de baño (Qd) de 800 $\mathrm{mL} / \mathrm{min}$, tiempo de diálisis de $269 \pm 32 \mathrm{~min}$, flujos de sangre $(\mathrm{Qb}) 443 \pm 29 \mathrm{~mL} / \mathrm{min}$, agujas del $15 \mathrm{G}$, peso $63,7 \pm 12 \mathrm{Kg}$., Hto $36,5 \pm 5 \%$, fraxiparina 0,325 \pm $0,16 \mathrm{ml}(1 \mathrm{ml}=950 \mathrm{UI}$ anti Xa).

El cebado del circuito en el sistema de mid-dilución es más laborioso que en las otras técnicas, ya que hay que hacer pasar un mínimo de 2 I de líquido de reinfusión, primero se ceba el haz central, y posteriormente las fibras periféricas. En la técnica de middilución el circuito sanguíneo inicialmente se realizó con entrada por el haz de fibras periféricas y retorno por el haz central. Sin embargo, en estudios posteriores, Santoro y col. observaron una menor presión intra-filtro con la infusión por el haz central de entrada y retorno periférico (Reverse Mid-dilución) ${ }^{4}$, por lo cual nosotros aplicamos esta forma de infusión en el estudio (figura 1 ).

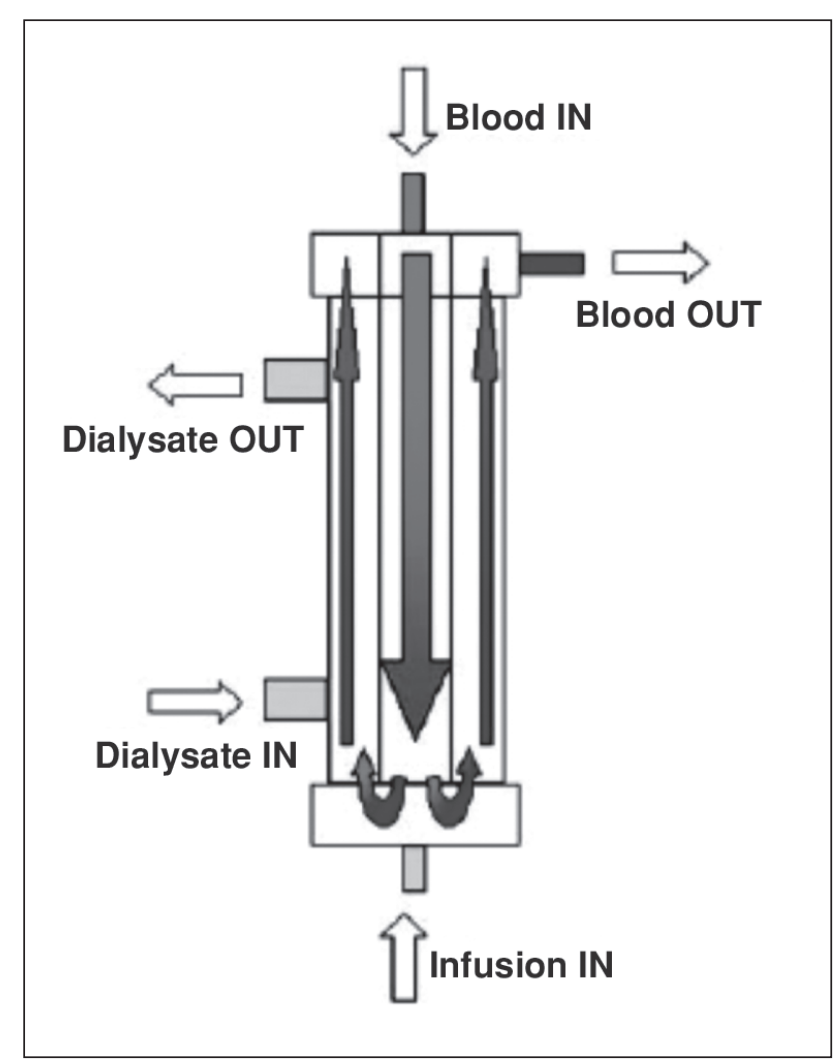

Figura 1. Filtro Reverse mid-dilution
Durante el estudio se registraron los valores de presión transmembrana (PTM) en la $1^{\text {a }}$ y $3^{\mathrm{a}}$ hora de hemodiálisis, el Kt en litros con dialisancia iónica, el volumen convectivo infundido, el peso inicial y final. Se realizaron analíticas pre- y post- sesión de diálisis para determinar los valores de urea, creatinina, $\beta_{2}$-microglobulina, mioglobina, prolactina y proteína transportadora de retinol (RBP), usados para calcular los porcentajes de reducción en cada sesión.

Los resultados se expresan como la media aritmética \pm desviación típica. Para el análisis de la significación estadística de parámetros cuantitativos se ha empleado ANOVA para datos pareados. Se ha considerado estadísticamente significativa una $p<0,05$.

\section{Resultados}

No se observaron complicaciones técnicas durante las sesiones, alcanzando los volúmenes de infusión pautados sin incidencias. Se redujo el número de incidencias de alarmas de presión venosa y PTM.

El volumen convectivo fue de $53,1,26,9$ y $52,9 \mathrm{~L}$ en el modo pre-, post- y mid-dilucional respectivamente. Los resultados de la PTM observados en la primera y tercera hora en las tres sesiones se muestran en la figura 2.

\section{PTM $1^{\text {a }}$ hora $(\mathrm{mm} \mathrm{Hg})$}
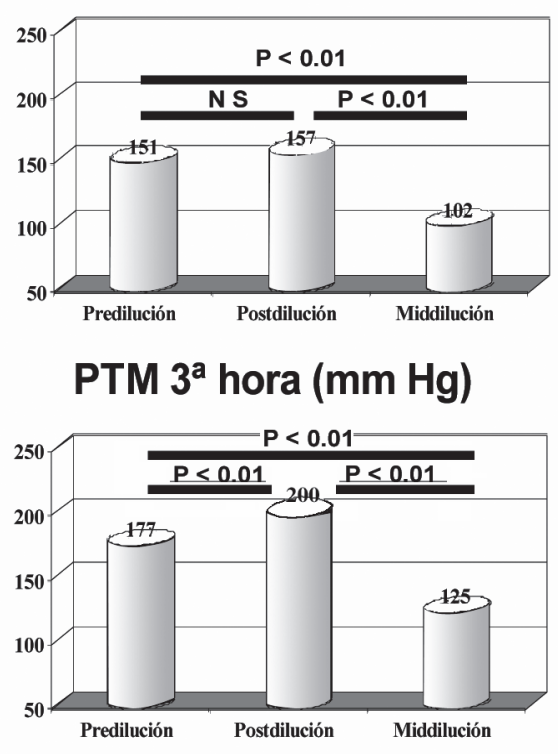

Figura 2. Presión transmembrana a la $1^{\mathrm{a}}$ y $3^{\mathrm{a}}$ hora 
Los porcentajes de reducción de urea y creatinina fueron ligeramente superiores en HDF post-dilucional frente HDF pre- y mid-dilucional (figuras 3 y 4 ).

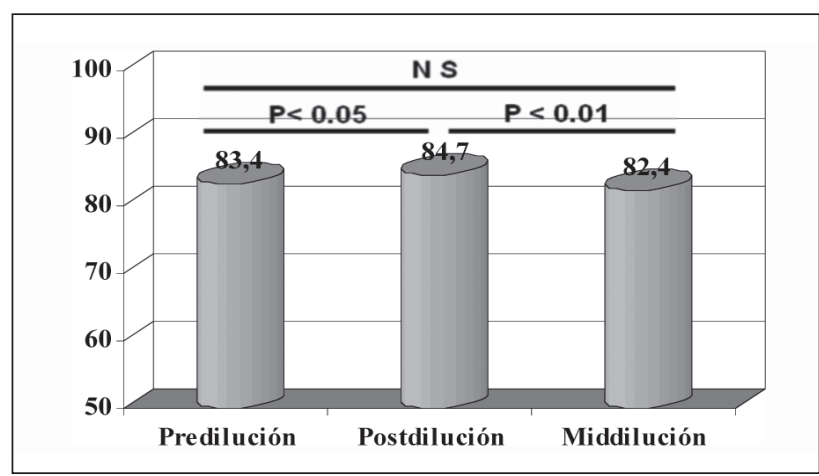

Figura 3. Porcentaje de la reducción de urea

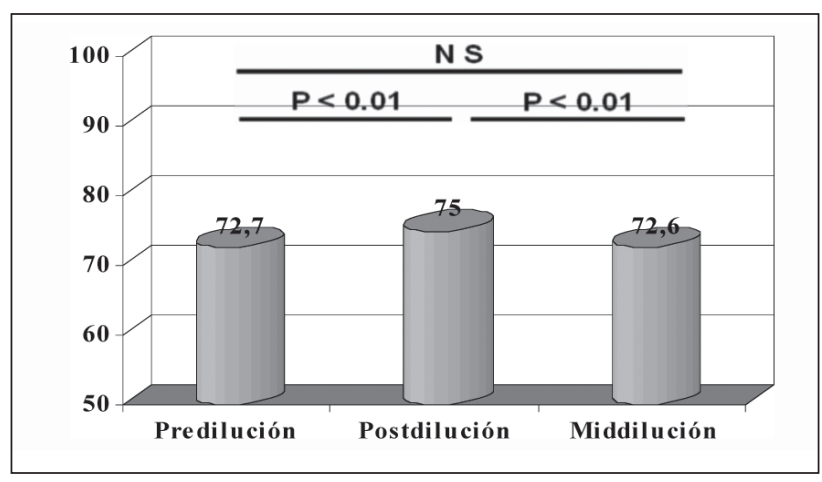

Figura 4. Porcentaje de la reducción de creatinina

Resultados similares de depuración de urea fueron obtenidos con dialisancia iónica y expresados como Kt en litros (figura 5).

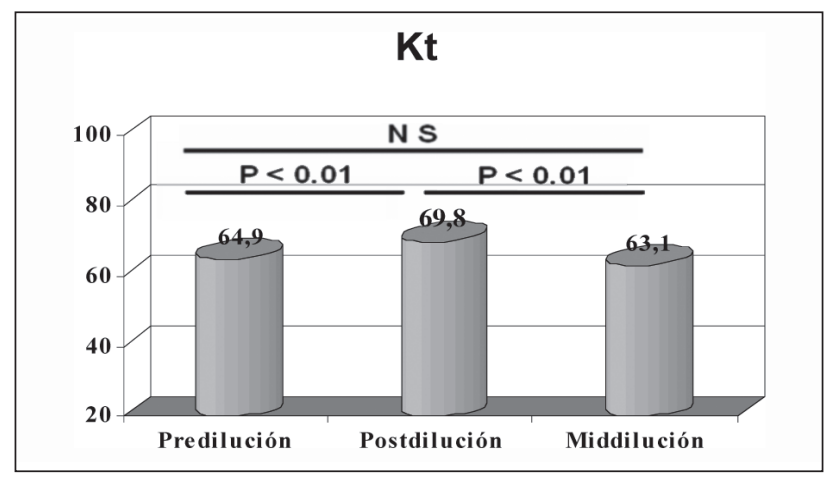

Figura 5. Depuración de urea por dialisancia iónica

Los porcentajes de reducción de $\beta_{2}$-microglobulina $(85,3 \pm 3 \%)$, mioglobina $(73,6 \pm 11 \%)$ y prolactina $(67,8 \pm 14 \%)$ en HDF mid-dilucional fueron significativamente superiores comparados con HDF post-dilucional $(83,3 \pm 3 \%, 68,4 \pm 10 \%$ y $62,8 \pm 8 \%$ respectivamente) y HDF pre-dilucional $(79,6 \pm 4 \%$, $54,9 \pm 12 \%$ y $51,8 \pm 10 \%$ respectivamente).

El porcentaje de reducción de RBP $(29,2 \pm 9 \%)$ con el modo HDF mid-dilucional fue significativamente superior al HDF post-dilucional $(23,5 \pm 10 \%, p<0.01)$ y HDF pre-dilucional $(22,5 \pm 10 \%, p<0.01)$.

\section{Discusión}

La eficacia depurativa es el objetivo principal en el que se centra el esfuerzo de investigación y desarroIlo de la diálisis. Esta demostrado que la diálisis HDF on-line es una técnica alternativa válida en el tratamiento de la insuficiencia renal crónica. La asociación entre transporte difusivo y convectivo garantiza buenos resultados en casos de solutos de peso molecular pequeño, medio y alto, permitiendo conseguir resultados depurativos adecuados.

Los métodos actuales de la diálisis HDF on-line más utilizados son la hemodiafiltración post- y pre-dilución, presentando ambos tanto características positivas como negativas 5 . La HDF pre-dilucional permite altos volúmenes de líquido de sustitución, elevados flujos de ultrafiltración y mejor convección con altos aclaramientos de moléculas de tamaño medio. El principal efecto negativo que presenta éste método es el bajo aclaramiento de las moléculas pequeñas, debido al bajo gradiente de concentración producido por la hemodilución.

La diálisis HDF post-dilucional favorece el aclaramiento de las moléculas de peso molecular pequeño y mediano. Puede presentar problemas de hemoconcentración con elevados niveles de PTM y limitación de la tasa máxima de reinfusión por debajo de los niveles normales (25-30\%) de flujo hemático. Las alarmas son más frecuentes, alargando el tiempo de sesión de la diálisis y aumentando la carga de trabajo de enfermería.

La diálisis HDF mid-dilución permite combinar las ventajas de las dos técnicas anteriores, minimizando los efectos negativos de éstas ${ }^{6}$. Reduce los niveles de hemoconcentración, consigue niveles de PTM dentro del rango, utiliza un gran volumen convectivo (200 $\mathrm{mL} / \mathrm{min}$ ), presenta buena tolerancia hemodinámica y 
mejora los resultados depurativos de la $\beta_{2}$-microglobulina, prolactina, mioglobina y RBP en comparación con los resultados de la post-dilucional, y aún mayores respecto a los pre-dilucionales.

En cuanto al trabajo de enfermería, se redujo el número de incidencias de alarmas de presión venosa y PTM. Las tensiones arteriales se mantuvieron estables durante toda la sesión, sin hipotensiones sintomáticas. No hubo presencia de residuos hemáticos y no fueron necesarios cambios de línea y dializadores, situación que ocasionalmente se produce en la postdilución. Aunque el sistema de cebado en este método es más laborioso, las ventajas que presenta en cuanto a la reducción de incidencias lo hacen un buen método de HDF on-line.

\section{Conclusiones}

La hemodiafiltración on-line con el modo de infusión mid-dilucional parece ser una buena alternativa que permite altos volúmenes convectivos con una mayor depuración de $\beta_{2}$-microglobulina, prolactina, mioglobina y RBP en comparación con el modo post-dilucional y más llamativa en comparación con HDF pre-dilucional.

La buena tolerancia clínica y disminución de incidencias durante la sesión de hemodiálisis, hacen de ésta técnica una buena opción tanto para el paciente como para el personal de enfermería.

\section{Bibliografía}

1. Maduell F: Hemodiafiltration. Hemodial Int 9: 4755, 2005.

2. Maduell F, García H, Hdez-Jaras J, Calvo C, Navarro V: Comparación de la infusión predilucional versus postdilucional en HDF en línea. Nefrología 1998; 18 (Suppl 3):49.

3. Maduell F. Optimizing the prescription of hemodiafiltration. Contrib Nephrol. Basel, Karger, 158: 225 231, 2007.

4. Santoro A, Ferramosca E, Mancini E, Monari C, Varasani M, Sereni L, Wratten M. Reverse mid-dilution: new way to remove small and middle molecules as well as phosphate with high intrafilter convective clearance. Nephrol Dial Transplant. 2007; 22(7):2000-5.

5. Pedrini L, De Cristofaro V, Pagliari B, Samà F: Mixed predilution and postdilution online hemodiafiltration compared with traditional infusion modes. Kidney Int 2000; 58(5):2155-2165.

6. Krieter DH, Falkenhain S, Chalabi L, Collins G, Lemke HD, Canaud B. Clinical cross-over comparison of mid-dilution hemodiafiltration using a novel dialyzer concept and post-dilution hemodiafiltration. Kidney Int. 2005; 67(1):349-56. 